\title{
Spectroscopy with Three-Dimensional Model Atmospheres of Late-Type Stars
}

\author{
Remo Collet \\ Research School of Astronomy and Astrophysics, Australian National University, \\ Cotter Rd, Weston Creek ACT 2611, Australia \\ email: remo.collet@anu.edu.au
}

\begin{abstract}
In this contribution, I present the results of a differential analysis of $\mathrm{CH}$ spectral lines for the determination of carbon abundances with three- and one-dimensional model atmospheres of late-type stars at various metallicities. 3D-1D abundance corrections are found to be significant particularly at low metallicities and for turn-off stars. The dependence of 3D-1D carbon abundance corrections on the stellar $\mathrm{C} / \mathrm{O}$ ratio is also discussed.
\end{abstract}

Keywords. stars: abundances, stars: late-type, stars: atmospheres, hydrodynamics, convection, radiative transfer, line: formation, stars: carbon

\section{Introduction}

Classical spectroscopic analyses of late-type stars usually rely on synthetic spectra computed with one-dimensional (1D) stationary hydrostatic model stellar atmospheres (e.g. Kurucz 1993; Gustafsson et al. 2008; Grupp 2004). Such models make use of approximate recipes such as the mixing-length theory (Böhm-Vitense 1958) for modelling convective energy transport near the stellar surface and lack a proper description of gas flows in stellar atmospheres.

In recent years, another class of model atmospheres based on three-dimensional (3D) radiation-hydrodynamic simulations of stellar surface convection has emerged (Nordlund et al. 2009; Freytag et al. 2012; Vögler 2004). In 3D simulations, the inhomogeneous temperature-density stratification of the gas at the stellar surface is modelled consistently with bulk gas flows, and convection arises naturally from the solution of the basic radiation-hydrodynamics equations without the need for additional approximate recipes. Because of their inherent differences in the basic physical assumptions, $1 \mathrm{D}$ and 3D models predict different temperature stratifications for the atmospheres of late-type stars, particularly at low metallicities (Asplund et al. 1999; Collet et al. 2007). When used for spectral synthesis calculations and spectroscopic abundance analyses, 3D model atmospheres can therefore lead to significantly different results compared with traditional analyses based on 1D models, especially for molecular lines (Collet et al. 2007; Dobrovolskas et al. 2010). In this contribution, I present the results of a differential analysis of weak $\mathrm{CH}$ lines at $4300 \AA$ for the derivation of carbon abundances with $3 \mathrm{D}$ and $1 \mathrm{D}$ models of late-type stars computed for different stellar parameters and metallicities.

\section{3D spectral line formation calculations: results and discussion}

I compute synthetic profiles for the CH $4300 \AA$ lines using a set of 3D STAGGER models atmospheres (Magic et al. 2013) of red giants, turn-off stars, and dwarfs with a range of solar scaled compositions with $[\mathrm{Fe} / \mathrm{H}]$ from +0.0 down to -4.0 as well as $1 \mathrm{D}$ models that 

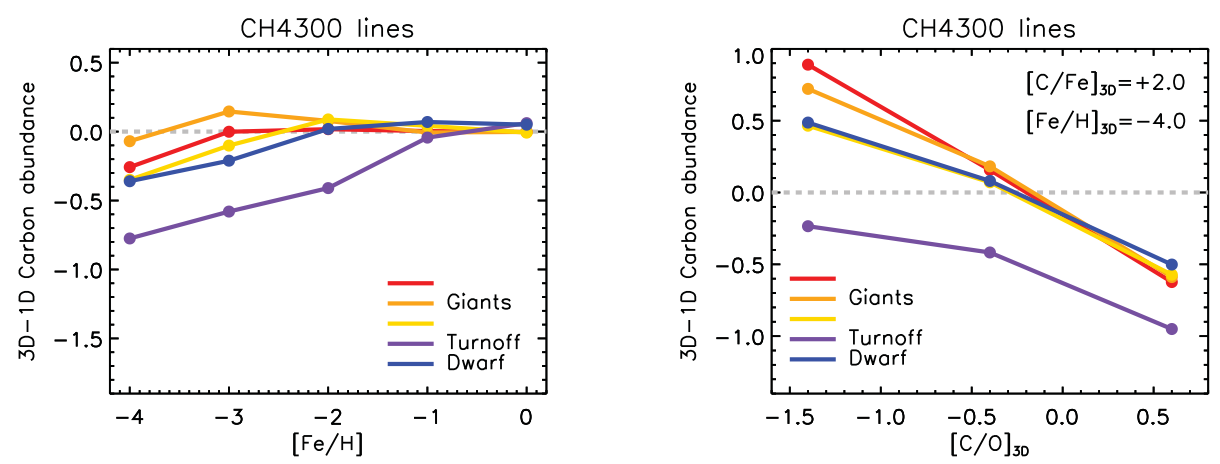

Figure 1. Left panel: 3D-1D carbon abundance corrections for late-type stars with different stellar parameters and metallicities. Right panel: carbon abundance corrections for CEMP stars with different atmospheric $\mathrm{C} / \mathrm{O}$ ratios.

were generated for exactly the same stellar parameters. I vary the $1 \mathrm{D}$ carbon abundance until the strength of the $3 \mathrm{D}$ line profiles is matched. I then compute the differences between the $3 \mathrm{D}$ and the so derived $1 \mathrm{D}$ abundances and refer to them as the $3 \mathrm{D}-1 \mathrm{D}$ carbon abundance corrections.

The left panel of Fig. 1 shows the 3D-1D abundance corrections computed for the adopted set of model atmospheres. Corrections vary considerably depending on the stellar parameters and tend to be largest at low metallicities and for turn-off stars. Oxygen and nitrogen abundance corrections derived from $\mathrm{OH}$ and $\mathrm{NH}$ lines at $3100 \AA$ and 3360 $\AA$, respectively, are also found to be systematically negative and more pronounced than for $\mathrm{CH}$ lines at low metallicities. Due to non-linearities and interdependencies among chemical species in the molecular equilibrium network, the number densities of various molecules hence the predicted strengths of molecular lines are sensitive to the atmospheric chemical composition. This is particularly relevant for carbon- and oxygenbased molecules whose number densities are strongly controlled by the carbon-to-oxygen $\mathrm{C} / \mathrm{O}$ ratio via $\mathrm{CO}$ molecule formation. Consequently, 3D-1D carbon abundance corrections are also a function of the $\mathrm{C} / \mathrm{O}$ ratio. The right panel of Fig. 1 shows that such corrections can vary dramatically with the value of the $\mathrm{C} / \mathrm{O}$ ratio in the atmosphere of a carbon-enriched metal-poor (CEMP) star. When evaluating the 3D-1D carbon abundance corrections for such objects it is therefore paramount to simultaneously and consistently determine the oxygen abundance in order to avoid large systematic errors.

\section{References}

Asplund, M., Nordlund, Å., Trampedach, R., \& Stein, R. F. 1999, AËA, 346, L17

Böhm-Vitense, E. 1958, Zeitschrift fur Astrophysik, 46, 108

Collet, R., Asplund, M., \& Trampedach, R. 2007, A\&SA, 469, 687

Dobrovolskas, V., Kucinskas, A., Ludwig, H. G., et al. 2010, in Nuclei in the Cosmos, 288

Freytag, B., Steffen, M., Ludwig, H.-G., et al. 2012, Journal of Computational Physics, 231, 919

Grupp, F. 2004, $A \mathscr{E} A$, 420, 289

Gustafsson, B., Edvardsson, B., Eriksson, K., et al. 2008, A\&์A, 486, 951 
Kurucz, R. 1993, CD-ROM No. 13. Cambridge, Mass.: Smithsonian Astrophysical Observatory Magic, Z., Collet, R., Asplund, M., et al. 2013, A\&\&A, 557, A26

Nordlund, A., Stein, R. F., \& Asplund, M. 2009, Living Reviews in Solar Physics, 6, 2

Vögler, A. 2004, A\&A, 421, 755 